Public Health

\section{Hearing and aging}

$\mathrm{C}$ linically significant hearing loss is the third most common condition among older adults. It is present in about $25 \%-40 \%$ of people over 65 years of age, $50 \%$ of those over 75 , and $80 \%$ of those over 85 years ${ }^{1}$ - becoming, with aging, the rule rather than the exception.

Of all our sensory modalities, hearing is the most integral to communication; hearing impairment therefore affects just about the entirety of human experience. Loss of hearing can damage one's mental processing, emotional and mental health, and educational and occupational prospects. Hearing loss, especially in older adults, also tends to lead to social isolation, depression, withdrawal from daily activities, and frustration with $\widehat{s}$ and among family members and friends.

\section{Types of hearing loss}

The schematic (Fig. I) shows the hearing system and the 2 types of hearing loss, conductive (referring to a defect in sound conductance to the inner ear) and sensorineural (caused by pathology in the inner ear, auditory nerves or other, more central structures). Agerelated hearing loss (presbycusis) is usually sensorineural. It refers to a functional decline in the ability to hear and process sound that is associated with aging and cannot be explained by the patient's genetic history, other diseases or trauma to the hearing system (e.g., mechanical trauma, noise and other agents such as toxic chemicals).

Although the degeneration with aging of the sensory cells in the cochlea, supporting cells and auditory nerve are well described, the exact cause of presbycusis is not known. Many older adults also experience degeneration of the central processes involved in decoding the auditory signal. Moreover, general cognitive function undergoes a decline with aging. Older adults tend to do worse than younger audiometrically matched people with difficult listening conditions such as occur in large rooms and other reverberant spaces (e.g., rooms with predominantly hard, sound-reflective surfaces), rapid speech, an unfamiliar speaker and competing background noise. The relative importance of degeneration of the peripheral and central mechanisms in presbycusis remains controversial.

\section{Screening for hearing loss}

Primary care professionals should think about hearing loss as a possible cause or contributing factor (among many) for social withdrawal, depression and reduced interaction with family members. Because presbycusis has a gradual onset, many older adults are unaware of their hearing problem; it may first be noticed by a caregiver or partner. It is common for affected individuals to deny their symptoms, at least initially.

Although many physicians lack access to equipment suitable for screening for hearing loss, it has been argued that prevalences are so high that all older adults should be tested ${ }^{2}$ with a screening device such as an audioscope (Welch Allyn, Skaneateles Falls, NY). Ultimately, everyone suspected of having a loss of hearing should undergo formal calibrated audiometry in a

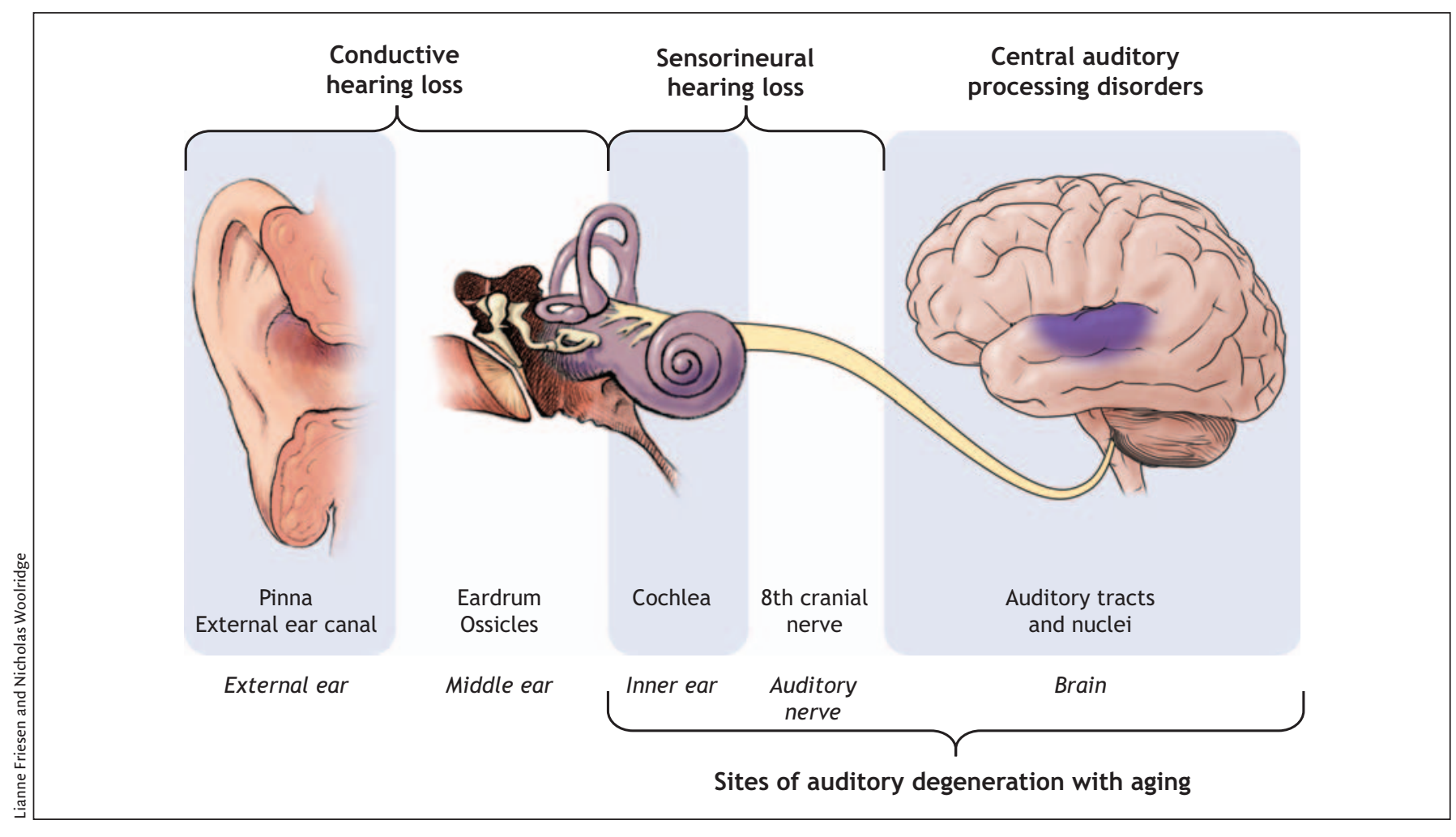

Fig 1: The hearing system. 
Table 1: Likelihood ratios for a hearing loss of a stated severity, if a patient passes or fails a given hearing test.

\begin{tabular}{lccc}
\hline & Hearing & \multicolumn{2}{l}{ Likelihood ratio } \\
\cline { 4 - 5 } Screening test & $\mathrm{dB}$ & Pass & Fail \\
\hline Single question, e.g., & $>25$ & 2.2 & 0.45 \\
“Do you have trouble & $>30$ & 2.4 & 0.49 \\
hearing?” & $>40$ & 2.5 & 0.13 \\
\hline HHIE-S score $>8$ & $>25$ & 4.5 & 0.55 \\
& $>40$ & 3.8 & 0.38 \\
\hline Whispered voice & $>30$ & 6.1 & 0.03 \\
\hline Audioscope* & $30-45$ & 2.4 & 0.07 \\
\hline
\end{tabular}

Note: HHIE-S = Hearing Handicap Inventory for the Elderly, screening version.

Information in this table was adapted from Bagai et $\mathrm{al}^{3}$ (JAMA 2006;295:416-28).

*Welch Allyn, Skaneateles Falls, NY.
Box 1: Questions in the Hearing Handicap Inventory for the Elderly, screening version

$$
\text { Overall scoring: yes }=4 \text { points, sometimes }=2 \text { points, no }=0
$$

Does a hearing problem cause you:

1. (E) To feel embarrassed when meeting new people?

2. (E) To feel frustrated when talking to members of your family?

3. (S) To have difficulty understanding, when someone speaks in a whisper?

4. (E) To feel handicapped by your hearing?

5. (s) To have difficulty when visiting friends, relatives or neighbours?

6. (S) To attend religious services less often than you would like?

7. (E) To have arguments with family members?

8. (s) To have difficulty when listening to television or radio?

9. (E) To feel that your hearing limits or hampers your personal or social life?

10. (s) To have difficulty when in a restaurant with relatives and friends?

Note: $(E)=$ emotional handicap question, $(S)=$ social handicap question. Adapted from Ventry and Weinstein (Ear Hear 1982;3:128-34). ${ }^{4}$ sound-attenuating booth with a trained audiologic professional - the "gold" standard for measurements of hearing loss. The performance of other screening measures are summarized in Table I, from Bagai and colleagues. ${ }^{3}$ Note that the whispered voice test, in my experience, can be highly variable, which limits its use as a screening test: results depend not only on how the test is carried out, but on background noise, as well.

Box I reproduces a widely used screening questionnaire, the Hearing Handicap Inventory for the Elderly (screening version). ${ }^{4}$ These questions also illustrate the types of situations in which people who are hard of hearing have problems.

In the end, if the patient or any caregiver or family member expresses a concern about hearing loss, or the patient, caregiver or a family member responds positively to a simple screening question (e.g., "Do you [or, Does he or she] have problems with hearing?"), formal audiometry testing is warranted. If the answer from the family member or caregiver as well as the patient is no, then rehabilitation options are unlikely to be followed through, even if the audiogram shows a hearing loss.

\section{Diagnosis of hearing loss}

Presbycusis, the predominant diagnosis in older adults, is a diagnosis of exclusion (Box 2). Hearing loss with aging can be thought of as the incremental sum of many otologic traumas acquired thoughout a lifetime, superimposed on the background of an intrinsic aging process. Of these, noise exposure is not only the most common cause but the one most difficult to separate from the aging process itself.

Wax impaction, also common in older adults, causes IO-I5 dB of hearing loss. With underlying presbycusis, this can have a noticeable effect on the ability to hear.

Box 2 and Box 3 highlight the important features of presbycusis, and the warning signs that warrant further investigation or referral to an otolaryngologist.

Important points to consider while taking the patient's history include asking about other causes of sensorineural hearing loss, such as ototoxic drugs (cisplatin, anti-inflammatories, some aminoglycosides), exposure to noise (both recreational and industrial), skull fractures, ear surgery, vertigo (associated with other inner-ear diseases) and a family history of hearing loss. The ears should be examined for wax; the middle ear, for perforations, effusion and other signs of middle-ear disease. New-onset middle-ear disease is uncommon among older adults; a firsttime effusion in the middle ear of an older adult requires an examination of the nasopharynx, to exclude lesions there. Tuning-fork tests are useful only for distinguishing conductive from sensorineural hearing loss.

\section{Rehabilitation of hearing loss}

There are no treatments to reverse presbycusis at present, but rehabilitation, usually including the use of hearing aids, can reduce the functional effects of hearing loss and improve quality of life.

Only about 10\%-20\% of those identified with hearing loss will become wearers of hearing aids. Hearing aids bring many challenges, and do not restore hearing to normal. Most audiologists will recommend bilateral hearing aids to improve the understanding of speech in crowded situations. Newer hearing aids have overcome many limitations (including feedback) and feature hand-held controls for changing settings easily (especially important for older adults) and multiple settings for different environments. Nevertheless, the dexterity problems of older adults can be a major factor in the choice of hearing-aid type.

Other assistive devices such as amplified telephones, television amplifiers and transmitters, and visual doorbell and fire alarm alerts are available. Some public places such as theatres or churches have wireless systems that provide a signal directly to the hearing aid.

For older adults whose hearing loss is severe to profound, cochlear implants are an option, just as they are for 
Box 2: Some characteristics of presbycusis

- This diagnosis is one of exclusion

- The hearing loss is usually sensorineural in type

- Hearing loss is symmetrical

- Individual progression rates vary widely

- In incidence it has no ethnic or gender predilection, but hearing loss progresses faster in men

- High frequencies are usually affected first, but the hearing loss can be "flat" across all frequencies

- It can coexist with other forms of hearing loss, such as those induced by wax buildup or noise

\section{Box 3: Indications for additional investigation of sensorineural hearing loss}

Further investigation is required when the hearing loss:

- Is asymmetric, with at least $15 \mathrm{~dB}$ difference from normal in at least 2 frequencies - use evoked potentials or MRI to rule out retrocochlear pathologies such as acoustic neuroma

- Is mostly restricted to low frequencies, which is more often associated with endolymphatic hydrops or other inner-ear diseases

- Is associated with attacks of vertigo or progressive ataxia - rule out inner-ear diseases such as Meniere's disease or autoimmune inner-ear disease

- Progresses rapidly (over weeks or months) - rule out ototoxicity, autoimmune inner-ear disease and rare systemic diseases such as Lyme disease and syphilis

- Has a conductive component - rule out external- and middle-ear problems such as wax buildup, eardrum perforation or cholesteatoma

- Occurs primarily at 3-4 kHz - rule out loud or continuous noise exposure, whether previous or ongoing (Note: such patients may be eligible for support from organizations such as the Workplace Safety and Insurance Board, in Ontario, or Veterans Affairs Canada) younger subjects. Most older people report levels of satisfaction similar to those of younger patients.

Ongoing counselling with an audiologic professional, which should include close family members, is critical to the successful use of hearing aids. The need for psychosocial adjustment makes it important to complement technological interventions with training and counselling. Even frail individuals can benefit from hearing aids or assistive technology if caregivers provide appropriate support.

\section{Future directions}

In animal studies, age-related hearing loss have been slowed with antioxi- dants, mitochondrial metabolites, restricted energy intake, neurotrophic factors, anti-apoptotic factors and auditory stimulation at lower volumes. These may ameliorate presbycusis, and one day even permit its reversal.

In terms of rehabilitation, newer hearing-aid options such as open mould fittings, implantable middle-ear ossicular drivers and new signal processing algorithms continue to improve the treatment options.

\section{Manohar Bance}

Division of Otolaryngology

Department of Surgery

Dalhousie University

Halifax, NS
This article has been peer reviewed.

Competing interests: None declared.

\section{REFERENCES}

I. Gates GA, Cooper JC Jr, Kannel WB, et al. Hearing in the elderly: the Framingham cohort, I983-1985. Part I: Basic audiometric test results. Ear Hear I990;1I:247-56.

2. US Department of Health and Human Services. Objectives for improving health (part B: Focus Areas I528). 28-I4 (Developmental). Increase the proportion of persons who have had a hearing examination on schedule. In: Healthy People 20Io, Volume II. 2nd ed. Washington; 2000 Jan. Available: www.healthy people.gov/Document/HTML/Volume2/28Vision.htm \#_Toc489325915 (accessed 2007 Feb 23).

3. Bagai A, Thavendiranathan P, Detsky AS. Does this patient have hearing impairment? JAMA 2006;295: 4I6-28.

4. Ventry IM, Weinstein BE. The Hearing Handicap Inventory for the Elderly: a new tool. Ear Hear 1982 ; 3:128-34.

\section{Canadian Adverse Reaction Newsletter \\ Bulletin canadien des effets indésirables}

To receive the Newsletter and health product Advisories free by email, join Health Canada's MedEffect mailing list.

Go to www.healthcanada.gc.ca/medeffect

Inscrivez-vous à la liste MedEffet de Santé Canada pour recevoir gratuitement par courriel le Bulletin et les Avis au sujet des produits de santé. Rendez-vous à l'adresse www.santecanada.gc.calmedeffet

Report adverse reactions toll free to Health Canada Signaler sans frais des effets indésirables à Santé Canada

Tel./Tél. : 866 234-2345• Fax/Téléc. : 866 678-6789 